\title{
Efficacy and Safety of Thermobalancing Therapy in Men with Lower Urinary Tract Symptoms/Benign Prostatic Hyperplasia was Confirmed by Clinical Trial
}

\author{
Simon Allen, M.D. Ph.D. \\ Director of Fine Treatment, United Kingdom, Inventor - Thermobalancing Therapy \\ info@finetreatment.com
}

\begin{abstract}
:
Background: Lower urinary tract symptoms/benign prostatic hyperplasia (LUTS/BPH) is the most common problem for men older than age 50, and in men older than 80 the prevalence from this disorder reaches $90 \%$. Benign tumor, by pressing the bladder and the urethra, causes LUTS that interfere men's quality of life. The aim of this 2.5-year investigation to find out if Thermobalancing therapy is effective and safe to treat LUTS/BPH

Methods: 124 men older than 55 with LUTS and the prostate volume $(P V)$ up to $60 \mathrm{~mL}$ received Thermobalancing therapy enabled by therapeutic device during 6 months. Before and after treatment were investigated the International Prostate Symptom Score (IPSS), including quality of life (QoL) index, ultrasound measurement of $P V$ and uroflowmetry maximum flow rate $\left(Q_{\max }\right)$. Also, before and after 7-month therapy the dynamics of the same parameters were studied in men with $\mathrm{BPH}$ and $\mathrm{PV}>60 \mathrm{~mL}$.

Results: After treatment the dynamics of clinical parameters in 124 men with $P V<60 \mathrm{~mL}$ and 2 men with $P V>$ $60 \mathrm{~mL}$ have shown positive results: the $P V$ level reduced $(P<0.001)$, increased Qmax $(P<0.001)$, improved $Q o L$ and diminished IPSS ( $P<0.001)$. The dynamics of the same measurements in the control-group who did not receive treatment have shown negative outcomes.

Conclusions: The observed positive effect of therapeutic device, termed Dr Allen's Device, for BPH has allowed us to recommend Thermobalancing therapy as a new safe physiotherapeutic solution for men with enlarged prostate. However, require time of the use of therapeutic device needs more investigations, though one point can be exposed now - the larger prostate needs longer therapy.
\end{abstract}

Keywords: enlarged prostate, bph treatment, prostate enlargement, thermobalancing therapy, prostate natural treatment, lower urinary tract symptoms, bph cause, dr allen's device, clinical trial.

\section{BACKGROUND}

According to The National Institute of Health, benign prostatic hyperplasia (BPH) is the most common prostate problem for men older than age 50. In 2010, as many as 14 million men in the United States had lower urinary tract symptoms suggestive of BPH, about $50 \%$ of men between the ages of 51 and 60 and up to $90 \%$ of men older than $80^{(1)}$. Current estimates are that the number of individuals 80 years and older in the US will rise from 9.3 million in 2000 to 19.5 million in 2030, an increase of over $100 \%^{(2)}$. BPH and bladder outlet obstruction (BOO) have substantial adverse effects on the public health in all counties, including India. Despite widespread use of medical therapy, BPH remains, on a population level, associated with a substantial incidence of BOO-associated adverse events, including LUTS, urinary infections, bladder calculi, urinary retention and acute renal failure (3).

Larger prostate volumes were positively associated with increased age. The total prostate volume $(\mathrm{mL})$ in average at $40-49$ years $=28$; at $50-59$ years $=30.8$; at 60-70 years $=35.0$. The mean length of prostate increased faster than the height and width, especially after the age of 60 years. These equations and models can facilitate further studies about prostate growth and may enable early diagnosis of $\mathrm{BPH}^{(4)}$.

Some experts have believed that hormones, particularly testosterone, to play a permissive role in the development of BPH/LUTS. This means that androgens have to be present for BPH to occur ${ }^{(5)}$. However castrated boys do not develop BPH when they age. In the following study of 26 eunuchs from the palace of the Qing dynasty still living in Beijing in 1960, the prostate was impalpable (could not be felt) in $81 \%{ }^{(6)}$. 
In the last decade, the pathogenesis of BPH began to consider from the perspective of vascular dysfunction, for instance, age might activate systemic vascular risk factors, resulting in disturbed blood flow ${ }^{(7)}$, furthermore, development of prostatic hyperplasia may be associated with prostatic hypoxia ${ }^{(8)}$, and with a correlation between pelvic ischemia and lower urinary tract symptoms in elderly men ${ }^{(9)}$, and also increasing the pressure in the prostate ${ }^{(10)}$.

Dr. Allen's research on the Origin of Diseases identifies the causal root of prostate enlargement, namely capillary expansion. This conclusion is based on 2 functional physiological properties of capillaries which are activated by an irritating factor, i.e. a trigger; indeed this peculiarity of capillaries explains the cause of chronic internal diseases. Constriction of capillaries in response to an irritating trigger develops local micro-hypothermia. It is this focus of hypothermia, which in turn becomes a constant irritant maintaining illness, i.e. making a disease chronic.

In response to irritation (i.e. a trigger-initiator and later focus of hypothermia) and in order to eliminate them, the blood flow increases through the spontaneous expansion of the capillary net locally. The formation of new capillaries is essentially the growth of the excess tissue that leads to an increased pressure inside the organ and its growth.

In other words, extra tissue becomes irritated as a response to different factors, in BPH event the initial trigger can be cold, stagnation of prostate own secretion or infection, and the secondary focus of hypothermia, both these factors lead to prostate growth. Thus, the cause of BPH based on vascular dysfunction. In order to terminate prostate growth and to reverse prostate enlargement, the improvement of blood circulation locally becomes the primary objective ${ }^{(11)}$.

Widely used BPH medications cannot reach the prostate tissue and provide symptomatically relief in its acute stage. Moreover BPH drugs and prostate surgeries may be responsible for different side effects ${ }^{(12)}$. For instance, the case studies published by the Harvard Medical School confirm complications arising from two BPH drugs: tamsulosin (Flomax) which may suddenly make men become lightheaded or develop retrograde ejaculation, and terazosin (Hytrin) which can be responsible for the acute urinary retention ${ }^{(13)}$. Dr. Irwig has confirmed persistence of erectile dysfunction after discontinuation of treatment with Propecia (finasteride). That is why a watchful waiting stage for BPH is recommended ${ }^{(14)}$.

The data above shows that in the etiology and pathogenesis of BPH vascular factor plays a decisive role, also BPH medications and surgeries are not safe and interfere men's quality of life. In order to improve men's wellbeing Thermobalancing therapy enabled by therapeutic device has been introduced. A 2-year controlled trial has shown the efficacy of thermobalancing therapy for men with $\mathrm{BPH}$ with prostate volume less than $60 \mathrm{~mL}^{(15)}$. In this study we also discuss the dynamics of the clinical symptoms and parameters in men with the prostate volume over $60 \mathrm{~mL}$.

\section{MAterials AND MethodS}

\subsection{Study Design}

The clinical controlled study was used. The Ethics Committee of the Yerevan State Medical University has approved the clinical study on Thermobalancing therapy enabled by therapeutic device. In the Department of Urology at the Yerevan State Medical University were compared men with BPH who received treatment with therapeutic device during 6 months and the control group on the watchful waiting. Dynamics of the symptoms and the indicators in each group were evaluated in comparison to their data in the beginning and end of the treatment.

\subsection{Evaluation}

The baseline evaluations included complete physical examination, medical history, DRE, serum biochemistry, and PSA measurements, electrolytes, urine and renal function tests. Evaluations were made at baseline and 6 months after the treatment. IPSS-QoL scored as follow: delighted $=0$, pleased $=1$, mostly satisfied $=2$, about equally satisfied and dissatisfied $=3$, mostly dissatisfied $=4$, hopeless $=5$, and poor $=6$. PV was measured at baseline and at 6 months after the treatment by ultrasonography (US-9000E2 ultrasound scanner, Rising Medical Equipment Co. Ltd, Beijing, China) and uroflowmetry (maximum urinary flow rate - Qmax, $\mathrm{mL} / \mathrm{s}$ ) was used for the measurement of the rate of urine flow parameters (Sanuro2UL, Santron Meditronic, Maharashtra, India). The standard ellipsoid formula length $\times$ width $\times$ height $\times 0.52$ was used to determine prostate volume.

\subsection{Participants and Interventions}

From 226 men with BPH 124 patients with prostate volume $(\mathrm{PV})<60 \mathrm{~mL}$ were selected for the clinical trial. 80 men were excluded, as their prostate volume was over $60 \mathrm{ml}$ or they had severe co- 
morbidities; 10 preferred operation; 4 were suspected prostate cancer; 8 did not attend to the following examinations. Men in treatment-group were given therapeutic device, termed Dr. Allen's Device, see figure 1.
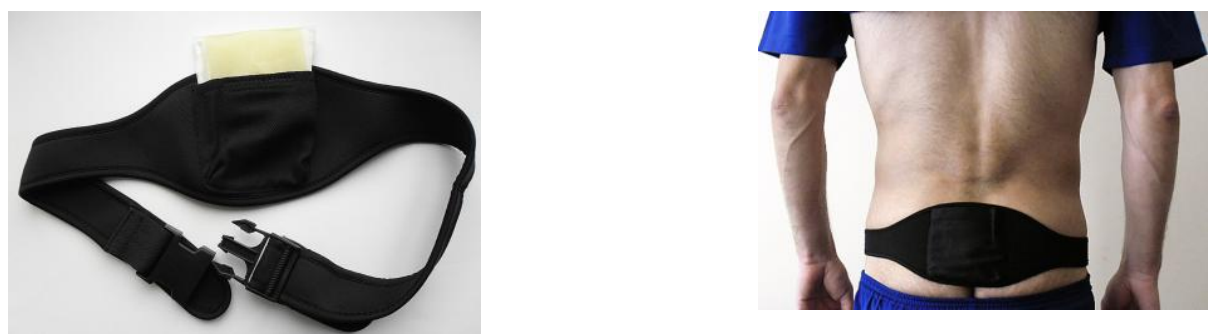

Figure1. The therapeutic device alone (on the left) and the device tightly applied to the coccyx area in the projection of the prostate.

\section{RESUlTS}

\subsection{Urinary Symptoms and QoL}

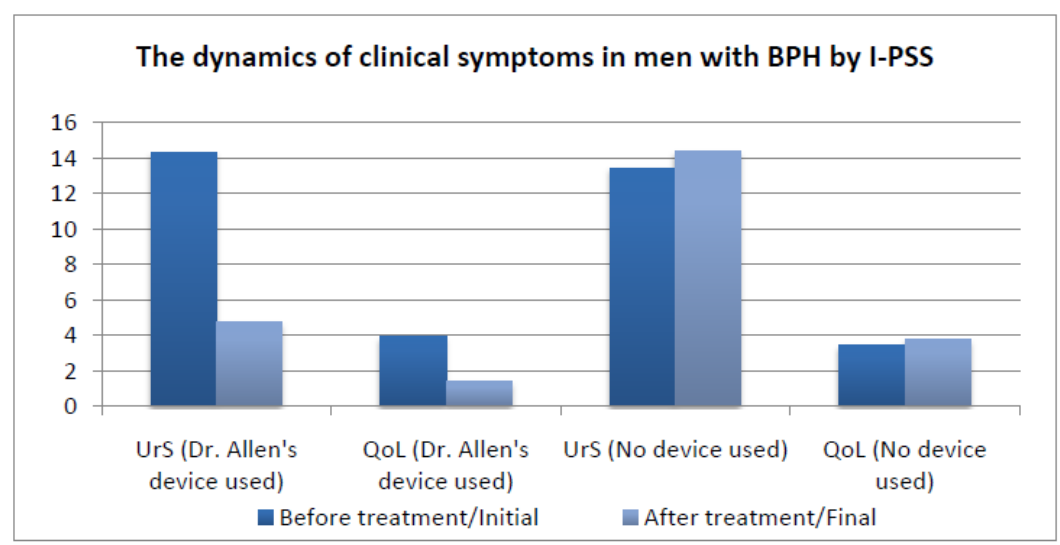

Figure2. International Prostate Symptom Score (IPSS), urinary symptoms (UrS) and quality of life (QoL), in patients with BPH on Thermobalancing therapy and in the control group at the beginning and at the end of the study.

In the control group, the mean IPSS-UrS increased from $13.45 \pm 3.254$ to $14.35 \pm 3.396$, whereas in the treatment group the mean IPSS-UrS decreased from $14.33 \pm 3.399$ to $4.73 \pm 2.754$ at the end of the observation period. For the control group, the $\mathrm{z}$ value was 6.018 with a $P$ value $(P<0.001)$. For the treatment group, the $\mathrm{z}$ value was 9.674 with a significance level $(P<0.001)$. This indicates that therapeutic device decreased the urinary symptoms significantly, while in absence of treatment the symptoms worsened significantly.

In the control group, the mean IPSS-QoL increases from $3.43 \pm 0.956$ to $3.76 \pm 0.983$, whereas in the treatment group the mean IPSS-QoL decreases from $3.91 \pm 0.755$ to $1.39 \pm 1.110$. For the control group, the $\mathrm{z}$ value was 5.286 with a $P$ value $(P<0.001)$. For the treatment group, the $\mathrm{z}$ value was 9.672 with a $P$ value $(P<0.001)$. These results indicated that the treatment with Therapeutic device improved the QoL while in the control group the QoL worsened.

\subsection{Prostate Volume and Qmax}

In the control group the mean prostate volume increased from 45.54 to $50.85 \mathrm{~mL}$, whereas in the treatment group the mean prostate volume decreased from 45.19 to $31.86 \mathrm{~mL}$. For the control group, the $\mathrm{z}$ value is -8.727 at the significance level $(P<0.001)$. So there was a statistically significant increase in the prostate volume in the control group. For the treatment group, the $\mathrm{z}$ value is -9.669 at the significance level $(P<0.001)$. So therapeutic device reduced PV significantly, whereas in the no treatment group the PV level increased.

In the control group, the mean Qmax decreased from $7.95 \pm 2.871$ to $7.7 \pm 2.695 \mathrm{~mL} / \mathrm{s}$, where as in the treatment group the mean Qmax increased from $8.10 \pm 3.041$ to $17.73 \pm 4.392 \mathrm{~mL} / \mathrm{s}$. For the control group, the $\mathrm{z}$ value was 1.929 and the $P$ value .054 (> .05), indicating no statistically significant difference. For the treatment group, the $\mathrm{z}$ value is 9.621 at the significance level $(P<0.001)$, indicating a significant increase in the Qmax. The results demonstrate that therapeutic device increased uroflowmetry Qmax significantly, whereas in the control-group - no significant difference in uroflowmetry Qmax. 


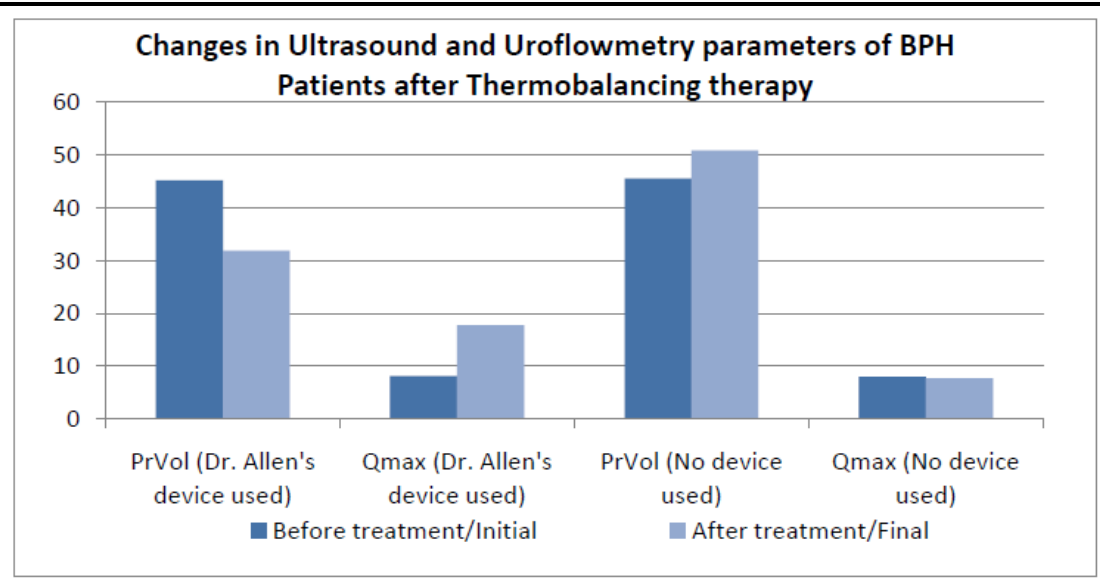

Figure3. The changes in prostate volume $(P V) m L$ and the uroflowmetry (maximum urinary flow rate (Qmax, $\mathrm{mL} / \mathrm{s}$ ) in men with BPH on Thermobalancing therapy and in the control group at the beginning and at the end of the study

\subsection{The Clinical Symptoms and Parameters in Men with BPH, $\mathrm{PV}>60 \mathrm{~mL}$}

Table1. The changes in prostate volume $(P V m L)$ and the uroflowmetry (maximum urinary flow rate $(Q m a x$, $\mathrm{mL} / \mathrm{s})$, Post Voiding Residual (ml) International Prostate Symptom Score (IPSS), in men with BPH (PV > 60 $m L)$ on Thermobalancing therapy

\begin{tabular}{|c|c|c|}
\hline Sargsyan $T$, age 73 & $01 / 07 / 2014$ & $03 / 11 / 2014$ \\
\hline IPSS - Urinary symptoms & 14 & 4 \\
\hline QoL index & 4 & 1 \\
\hline Prostate volume $\mathrm{mL}$ & 93 & 70 \\
\hline Q-max mL/s & 11.2 & 14.0 \\
\hline Nersesyan Telman age 79 & $22 / 07 / 2014$ & $18 / 02 / 2015$ \\
\hline IPSS & 6 & 2 \\
\hline QoL index & 3 & 1 \\
\hline Prostate volume $\mathrm{mL}$ & 154 & 90 \\
\hline Q-max mL/s & 13.0 & 14.4 \\
\hline Galstyan L, age 75 & $03 / 2 / 2014$ & 03.11 .2014 \\
\hline IPSS - Urinary symptoms & 12 & 3 \\
\hline QoL score & 4 & 0 \\
\hline Prostate volume $\mathrm{mL}$ & 84 & 52 \\
\hline Q-max mL/s & 9.7 & 15.5 \\
\hline Ambartsumyan $R$, age 73 & $18 / 03 / 2014$ & $10 / 03 / 2015$ \\
\hline IPSS - Urinary symptoms & 16.5 & 6 \\
\hline QoL score & 3 & 1 \\
\hline Prostate volume $\mathrm{mL}$ & 74 & 51 \\
\hline Q-max mL/s & 7.2 & 10.3 \\
\hline
\end{tabular}

These cases show the age of all men with BPH, PV $>60 \mathrm{~mL}$, was over 70. Therapeutic device used as a long-term mono therapy that decreased PV and increased Q-max, consequently was observed improvement in IPSS and QoL. Despite the fact that the PV decreased significantly in all cases, the size of the prostate remained large. This means that these patients should use the device longer.

\section{DisCuSSION}

This study results have demonstrated that Thermobalancing therapy decreased IPSS, reduced PV, increased the uroflowmetry Qmax and improved QoL in main treatment group 124 men with BPH, $\mathrm{PV}<60 \mathrm{~mL}$, and in 4 men with $\mathrm{BPH}$, PV $>60 \mathrm{~mL}$. These positive outcomes indicate that thermobalancing therapy is effective for BPH.

BPH is historically believed to be a consequence of the aging process and the elimination of the negative impact of an enlarged prostate in men should be done with the help of medical or surgical intervention. At the same time, medications used for the treatment of BPH do not provide sufficient performance. For example, a study showed that half (52.8\%) of men with BPH were dissatisfied with the results of medical treatment conducted according to current international guidelines for BPH ${ }^{(16)}$.

In addition, most commonly used BPH medications have side effects, especially in the long-term use ${ }^{(17)}$. Surgical treatment of prostate may also be accompanied by new challenges. Thus, the results of a survey of sexually active men after three different laser surgeries from 2005 to 2010, concluded that 
these surgical techniques can have a negative impact on sexual function, and patients with normal preoperative sexuality are more at risk ${ }^{(18)}$.

In the last decade, the opinion of the necessity of medical/surgical treatment of BPH has been challenged. BPH/LUTS should not be viewed as an inevitable disease of older people but part of the aging process which can be prevented ${ }^{(19)}$. In the last decade many studies suggest that NSAIDs have potential to improve symptoms and reduce the risk of prostatic diseases ${ }^{(20)}$. The evidence suggests that NSAIDs improve urinary symptoms and flow measures. Their long-term effectiveness, safety and ability to prevent BPH complications are not known ${ }^{(21)}$. However, non-selective non-steroidal antiinflammatory drugs (NSAIDs) should be used with caution especially in older people after other safer treatments have not provided sufficient pain relief. The lowest dose should be provided, for the shortest duration. It important as at age 55 overall lifetime risk of cardiovascular disease was $67.1 \%$ ${ }^{(22)}$. Thus, all older people taking NSAIDs should be routinely monitored for gastrointestinal, renal and cardiovascular side effects, and drug-drug and drug-disease interactions ${ }^{(23)}$.

Thermobalancing therapy can play a crucial role in the prevention of BPH development, particularly for moderate to low degree of LUTS secondary to prostate enlargement ${ }^{(24)}$. Thermobalancing therapy is different than heating treatments, as the source of energy doesn't exceed the normal body temperature range. Treatments with imposed heat can be damaging, because the high temperatures above 104 Fahrenheit (or 40C) destroy the living organism. On the other side, low temperatures decrease cellular metabolism.

Therapeutic device applies the thermoelement tightly to the skin overcoming the skin barrier and spreading energy inside toward the prostate, there is no other treatment method that is able to overcome the skin barrier delicately and precisely. Thermobalancing therapy is the only external noninvasive treatment that targets the pathological point of origin continuously for a prolonged period of time, i.e. for days, months or even years. I believe that the use of therapeutic device by keeping the body temperature in the projection of the prostate gland acts on micro-focus of hypothermia and ischemia, removing the vicious cycle of spontaneous growth of capillaries in response to triggers, thereby relieving the BPH symptoms.

\section{Conclusions}

This study has confirmed the effectiveness and safety of Thermobalancing therapy for the conservative treatment of moderate to low degree of LUTS secondary to prostate enlargement. The investigations in men with $\mathrm{BPH}, \mathrm{PV}<60 \mathrm{~mL}$, and in 4 men with $\mathrm{BPH}, \mathrm{PV}>60 \mathrm{~mL}$ after the use therapeutic device topically support the concept that hypothermia and changes in blood microcirculation play a role in the development of BPH/ LUTS. Thus, Thermobalancing therapy can be a new opportunity for secure effective physiotherapeutic intervention in $\mathrm{BPH}$. The significant reduce of prostate volume under Thermobalancing therapy makes necessity to study the changes in the prostate gland precisely, possibly by using a magnetic resonance imaging (MRI).

\section{Safety and Costs}

Patients feel no side effects during the clinical trial. Besides all of this, the important factor is the cost of a new treatment compared to the cost of the standard treatment options.

\section{Author Contributions}

Both authors made substantive intellectual contributions to the presented study. They have made substantial contributions to the conception and design and to the acquisition and interpretation of data, have been involved in drafting the manuscript and revising it critically for important intellectual content, and have given final approval of the version to be published.

\section{Conflicts of Interest}

The authors declare that they have no competing interests. Simon Allen applied to USPTO in 2009"Therapeutic Device and Method"- but has not received reimbursements, fees, funding, or salary relating to the content of the manuscript.

\section{REFERENCES}

[1] Deters LA. Benign prostatic hypertrophy. Emedicine website. http://emedicine.medscape.com/ article/437359-overvie.

[2] Centers for Disease Control and Prevention (CDC). Trends in aging-United States and worldwide. MMWR Morb Mortal Wkly Rep. 2003; 52(6):101-4,106. 
[3] Patel ND, Parsons JK Epidemiology and etiology of benign prostatic hyperplasia and bladder outlet obstruction, Indian J Urol. 2014 Apr; 30(2):170-6.

[4] Shi-Jun Zhang, Hai-Ning Qian, Yan Zhao et al, Relationship between age and prostate size, Asian J Androl. 2013 Jan; 15(1): 116-120.

[5] Bhasin S, Singh AB, Mac RP et al, Managing the Risks of Prostate Disease During Testosterone Replacement Therapy in Older Men: Recommendations for a Standardized Monitoring Plan, Journal of Andrology, 2003, 24, 3, 299-311.

[6] Wu CP, Gu FL, The prostate in eunuchs, Prog Clin Biol Res. 1991;370:249-55.

[7] Shimizu S, Tsounapi P, Shimizu $\mathrm{T}$, et al, Lower urinary tract symptoms, benign prostatic hyperplasia/benign prostatic enlargement and erectile dysfunction: Are these conditions related to vascular dysfunction? Int J Urol, 21(9):856-64, September 2014.

[8] Saito M, Tsounapi P, Oikawa $\mathrm{R}$ et al Prostatic ischemia induces ventral prostatic hyperplasia in the SHR; possible mechanism of development of BPH, Scientific Reports, 2014, doi: 10.1038.

[9] Thurmond P, Yang JH, Azadzoi KM LUTS in pelvic ischemia: a new concept in voiding dysfunction, Am J Physiol Renal Physiol. 2016 Jan 20:ajprenal.00333.2015.

[10] Cohen PG, Abdominal obesity and intra-abdominal pressure: a new paradigm for the pathogenesis of the hypogonadal-obesity-BPH-LUTS connection, Hormone Molecular Biology and Clinical Investigation, 2012, 11-1, 317-320.

[11] Allen S, Adjani a Therapeutic Device and Method, US 20110152986 A1, 2009, http://www.google.com/patents/US20110152986.

[12] Ben-Zyi T, Hueber PA, Valdivieso R, et al, Urological resident exposure to transurethral surgical options for BPH management in 2012-2013: A pan-Canadian survey, Can Urol Assoc J. 2014 Jan-Feb; 8(1-2): 54-60.

[13] Harvard Med School, Prostate Knowledge, 2007, your benign prostatic hyperplasia medication: When to consider a change, http://www.harvardprostateknowledge.org/your-benign-prostatichyperplasia-medication-when-to-consider-a-change.

[14] Irwig MS, Kolukula S Persistent sexual side effects of finasteride for male pattern hair loss, J Sex Med. 2011 Jun;8(6):1747-53.

[15] Allen S, Aghajanyan IG Benign Prostatic Hyperplasia Treatment with New Physiotherapeutic Device, Urol J. 2015 Nov 14; 12(5):2371-6.

[16] Fourcade RO, Lacoin F, Rouprêt M, et al. Outcomes and general health-related quality of life among patients medically treated in general daily practice for lower urinary tract symptoms due to benign prostatic hyperplasia. World J Urol. 2012; 30:419-26.

[17] Gacci M, Ficarra V, Sebastianelli A, et al. Impact of Medical Treatments for Male Lower Urinary Tract Symptoms Due to Benign Prostatic Hyperplasia on Ejaculatory Function: A Systematic Review and Meta- Analysis. J Sex Med. 2014; 11:1554-66.

[18] Elshal AM, Elmansy HM, Elkoushy MA, Elhilali MM. Male sexual function outcome after three laser prostate surgical techniques: a single center perspective. Urology. 2012; 80:1098-104.

[19] Corona G, Vignozzi L, Rastrelli G, Lotti F, Cipriani S, Maggi M. Benign Prostatic Hyperplasia: A New Metabolic Disease of the Aging Male and Its Correlation with Sexual Dysfunctions. Int J Endocrinol. 2014; 2014:329456.

[20] Ishiguro H, Kawahara T Nonsteroidal Anti-Inflammatory Drugs and Prostatic Diseases BioMed Research International 2014 436123:6.

[21] Kahokehr A, Vather R, Nixon A, et al Non-steroidal anti-inflammatory drugs for lower urinary tract symptoms in benign prostatic hyperplasia: systematic review and meta-analysis of randomized controlled trials, BJU Int. $2013 \mathrm{Feb} ; 111(2): 304-11$.

[22] Leening MJG, Ferket BS, Steyerberg EW et al Sex differences in lifetime risk and first manifestation of cardiovascular disease: prospective population based cohort study, BMJ 2014; 349:g5992.

[23] Abdulla A, Adams N, Bone M Guidance on the management of pain in older people, Age Ageing. 2013 Mar; 42 Suppl 1:i1-57.

[24] Aghajanyan IG, Allen S Positive Response to Thermobalancing Therapy Enabled by Therapeutic Device in Men with Non-Malignant Prostate Diseases: BPH and Chronic Prostatitis, Diseases 2016, 4, 18. Doi: 10.3390/diseases4020018. 